\title{
Vitamin $D$ intakes of adults differ by income, gender and race/ethnicity in the USA, 2007 to 2010
}

\author{
Carolyn E Moore ${ }^{1, *}$, John D Radcliffe ${ }^{1}$ and Yan Liv ${ }^{2}$ \\ 'Department of Nutrition and Food Sciences, Texas Woman's University, 6700 Fannin, Houston, TX 77030, \\ USA: ${ }^{2}$ US Department of Agriculture/Agricultural Research Service, Children's Nutrition Research Center, \\ Department of Pediatrics, Baylor College of Medicine, Houston, TX, USA
}

Submitted 20 December 2012: Final revision received 25 September 2013: Accepted 30 September 2013: First published online 31 0ctober 2013

\begin{abstract}
Objective: To determine if dietary, supplemental and total vitamin D intakes in the USA are influenced by income, race/ethnicity or gender.

Design: Cross-sectional. US vitamin D intakes were estimated by poverty income ratio (PIR), race/ethnicity and gender using $24 \mathrm{~h}$ dietary intake recalls and dietary supplement use questionnaires. Statistical analyses of weighted data were performed using SAS (version 9.2) to estimate means and their standard errors. Race and ethnic intake differences controlling for PIR, gender and age were assessed by ANCOVA.

Subjects: Adults aged $\geq 19$ years.

Setting: The 2007-2010 National Health and Nutrition Examination Survey, USA. Results: Total (dietary and supplement) vitamin D intake was greater in the high $(10 \cdot 0(\operatorname{se} 0.30) \mu \mathrm{g} / \mathrm{d}) v$. the medium $(7 \cdot 9$ (SE 0.3$) \mu \mathrm{g} / \mathrm{d}$ ) or the low (8.0 (SE 0.3) $\mu \mathrm{g} /$ d) PIR categories. Total vitamin D intake of non-Hispanic Whites (10.6 (sE $0 \cdot 4$ ) $\mu \mathrm{g} / \mathrm{d}$ ) was greater than that of Hispanics $(8 \cdot 1$ (sE $0 \cdot 3) \mu \mathrm{g} / \mathrm{d}$ ) and non-Hispanic Blacks $(7 \cdot 1($ SE $0 \cdot 3) \mu \mathrm{g} / \mathrm{d})$. Supplemental vitamin D intake was greater by females $(5 \cdot 3(\operatorname{SE} 0 \cdot 2) \mu \mathrm{g} / \mathrm{d})$ than by males $(3 \cdot 3(\mathrm{SE} 0 \cdot 2) \mu \mathrm{g} / \mathrm{d}$ ). Participants with high income were more likely to be vitamin D supplement users (33.0\%) than those with medium $(22.5 \%)$ or low $(17.6 \%)$ income. High-income non-Hispanic Whites had the lowest percentage (57\%) not meeting the Estimated Average Requirement for vitamin D. Fortified milk and milk products provided $43.7 \%$ of the dietary vitamin $\mathrm{D}$ intake.

Conclusions: Public health efforts should expand the number of vitamin D-fortified foods and encourage the consumption of foods high in vitamin D and use of supplements.
\end{abstract}

Vitamin D status has become a public health concern in the USA over the last several years due to an increasing number of reports of vitamin D deficiency. Recently, the identification of vitamin $\mathrm{D}$ receptors in most tissues indicates an expanded role of vitamin $\mathrm{D}$ beyond the classic actions of maintaining bone health. Vitamin D may provide a protective effect against CVD and cancer ${ }^{(1)}$. High serum vitamin D levels among adult populations are associated with a substantial decrease in CVD, type 2 diabetes and the metabolic syndrome ${ }^{(2)}$. Multiple factors contribute to health disparities, and vitamin D status may play a role in differences in the disease and mortality rates between Blacks, Hispanics and Whites in the USA ${ }^{(3)}$. Utilizing the 2005-2006 National Health and Nutrition Examination Survey (NHANES) data and a definition of vitamin $\mathrm{D}$ deficiency based on serum 25 -hydroxyvitamin $\mathrm{D}$ (25(OH)D) concentrations $\leq 20 \mathrm{ng} / \mathrm{ml}$, the overall prevalence rate of vitamin $\mathrm{D}$ deficiency was estimated to be
$41.6 \%$ for US adults, with the highest rate seen for Blacks $(82 \cdot 1 \%)$, followed by Hispanics $(69 \cdot 2 \%)$ and Whites $(30 \cdot 0 \%)^{(4)}$. Differences in vitamin D status, therefore, may contribute to health disparities between race/ethnic groups and influence the risk of some of the leading causes of death in the USA ${ }^{(5)}$.

The two major forms of vitamin $\mathrm{D}$ are ergocalciferol (vitamin $\mathrm{D}_{2}$ ) and cholecalciferol (vitamin $\mathrm{D}_{3}$ ). Cholecalciferol occurs naturally in very few foods (oily fish, meat, egg yolks) and is added to some foods (milk, milk products, calciumfortified juices, fortified breakfast cereals). Ergocalciferol is produced in mushrooms containing ergosterol when exposed to UV radiation and is added to foods such as soya milk. Fortified foods represent the major dietary source of vitamin $\mathrm{D}^{(6)}$ and more than one-half of the US population uses dietary supplements ${ }^{(7)}$. In addition, vitamin $\mathrm{D}$ is produced endogenously through exposure of skin to UV light, which stimulates conversion of the 
precursor 7-dehydrocholesterol to pre-vitamin $\mathrm{D}_{3}$ that is rapidly converted to cholecalciferol ${ }^{(8)}$.

In 1991, the US Department of Agriculture included the vitamin D content of foods for the first time in the National Nutrient Database for Standard Reference (SR). Vitamin D concentrations were obtained from some analytical data, processed food label declarations and calculated values from ingredient composition ${ }^{(9)}$. In 2008, HPLC and UV spectroscopic detection were used to measure ergocalciferol and cholecalciferol concentrations of milk, soya milk, cheese, calcium-fortified juices and breakfast cereals. The new analytical data were added to SR22 and used to estimate vitamin D intakes for the NHANES 2005-2006 cycle $^{(10-12)}$. Since then, as vitamin D-fortified products (yoghurts, margarine brands, cereal bars, etc.) enter the marketplace ${ }^{(13)}$, vitamin D content is routinely measured by HPLC and UV spectroscopic detection and incorporated into SR updates.

In 1997, the Institute of Medicine released Adequate Intakes for vitamin $\mathrm{D}$ rather than RDA because there was insufficient scientific evidence to determine an Estimated Average Requirement (EAR) ${ }^{(14)}$. EAR are used in surveillance studies to compare population intakes with recommended intakes of healthy people ${ }^{(14)}$ and serve as the nutrient-based reference values for setting the RDA. In 2010, EAR and RDA for vitamin D were established because more extensive information and higher-quality studies were finally available ${ }^{(15)}$.

Many investigators concluded that Blacks and Hispanics were at higher risk of vitamin D deficiency than Whites ${ }^{(16-20)}$. Multiple factors likely contributed to race/ethnic vitamin D differences including economic status, reduced nutrient intake and the skin pigmentation. Minority groups are more likely to have lower incomes compared with Whites ${ }^{(21)}$ and their access to nutritious food may be too expensive or limited, therefore contributing to nutrient health disparities ${ }^{(22)}$. In addition, increased skin pigmentation inhibits cutaneous synthesis of vitamin D and potentially increases the risk of a deficiency for darker-skinned individuals ${ }^{(23,24)}$.

Given the increased interest in the role of vitamin D in health, the continuing vitamin D analytical updates of the food supply, the establishment of EAR and RDA levels for vitamin D in 2010 and the interest in economic and race/ ethnic factors that may contribute to health disparities, we estimated vitamin D intake in the USA for adults aged $\geq 19$ years during 2007-2010. We then compared total, dietary and supplemental vitamin $\mathrm{D}$ intakes by poverty income ratio (PIR), race/ethnicity and gender. Finally, we identified the major foods groups in 2007-2010 contributing to the dietary intake of vitamin D.

\section{Methods}

\section{Survey design and source of data}

The NHANES is a cross-sectional surveillance programme conducted on a continual basis by the National Center for
Health Statistics of the Centers for Disease Control and Prevention that is released in 2-year increments to allow examination of the relationship between diet, nutrition and health ${ }^{(25)}$. The survey uses a complex multistage probability sampling design with oversampling of certain races/ethnicities and age groups ${ }^{(26)}$. Data were collected by trained NHANES personnel using the multiple-pass method $^{(27)}$ during in-household interviews as a part of the household and mobile examination centre interviews. A detailed description of the survey design, content, operations and procedures can be found elsewhere ${ }^{(28)}$.

For the present analyses, 11857 adults aged $\geq 19$ years participating in the 2007-2008 and 2009-2010 NHANES cycles were combined to increase sample size. The study measures obtained from the NHANES demographic file included PIR, race/ethnicity, gender and age. Of the original NHANES 2007-2010 data set, participants were excluded if PIR information was missing or if $24 \mathrm{~h}$ dietary recall data were judged to be incomplete or unreliable by the Food Surveys Research Group ${ }^{(29)}$. Also excluded from the analyses were 172 women who were pregnant or lactating and six participants taking large dosages of vitamin D supplements ( $\geq 1250 \mu \mathrm{g} / \mathrm{d}$ ).

Ethnicity and race were derived from self-reported information obtained in the screener and the household interviews ${ }^{(30)}$. For our analyses, groups were categorized into the following: non-Hispanic (NH) Whites, nonHispanic (NH) Blacks and Hispanics. Other ethnic groups were not included due to the small sample size. Hispanics were comprised of Mexican American and Other Hispanic combined into one Hispanic group to increase sample size for analysis.

Participants were classified as males or females based on self-reported data and categorized into income groups by PIR which was determined by the US Census Bureau method of dividing family income by poverty threshold specific to a family size ${ }^{(31)}$. Participants were classified into low income ( $\leq 131 \%$ of PIR), medium income $(>131 \%$ to $\leq 185 \%$ of PIR) or high income ( $>185 \%$ of PIR).

The study was conducted according to the guidelines laid down in the Declaration of Helsinki. All procedures in the 2007-2010 NHANES were approved by the National Center for Health Statistics Ethics Review Board and written informed consent was obtained from all participants ${ }^{(28)}$.

\section{Dietary assessment}

Dietary intake data were obtained from in-person $24 \mathrm{~h}$ dietary recall household interviews administered using an automated multiple-pass method at the mobile examination centre ${ }^{(27)}$. During the household interview, a Dietary Supplement Questionnaire was completed to collect information regarding the use of vitamins, minerals, herbs and other dietary supplements. Descriptions of the dietary interview methods are provided in the NHANES Dietary Interviewer's Training Manual ${ }^{(32)}$. 


\section{Covariates}

Food group consumption tends to vary by demographic and economic status; therefore, demographic characteristics (i.e. race/ethnicity, gender and age) and economic status (i.e. PIR) were considered as potential covariates in the analyses. The data for all covariates were obtained from the dietary recall and questionnaires.

\section{Estimates of vitamin D intakes from food sources}

The intake of vitamin D from food sources was determined for the major food categories used by the Food and Nutrient Database for Dietary Studies ${ }^{(33)}$. In addition, the proportion of intake of the major food categories was assessed by income. Within the major food groups, vitamin D intake was estimated by food sub-categories.

\section{Estimates of vitamin $D$ adequacy}

The percentage of the population during 2007-2010 not meeting the EAR and the RDA from dietary or total intake was estimated for the total population by income and age group.

\section{Statistical analyses}

Four-year sample weights and weighted data were calculated for all statistical analyses to account for the complex sample design, which was multistage, stratified, unequally weighted or clustered. Statistical analyses were performed using the statistical software package SAS version 9.2. PROC SURVEYFREQ and PROC SURVEYMEANS and procedures of SAS were used to produce the estimated frequencies of categorical variables, presented as frequency percentage and standard error, for all categorical measures with the Wald $\chi^{2}$ test and the means for continuous variables, being presented as mean and standard error.

For evaluating differences in vitamin D intake across the three race/ethnic groups, least-square means and standard errors were compared by ANCOVA using PROC SURVEYREG of SAS, controlling for age, gender or PIR. A $P$ value of $\leq 0.05$ was used to determine statistical significance and the $t$ test with Tukey-Kramer adjustment was used for multiple comparisons.

\section{Results}

A total of 9719 participants not taking high dosages of vitamin D supplements from NHANES 2007-2010 provided complete demographic and in-person $24 \mathrm{~h}$ dietary recalls determined to be reliable and to meet overall quality and completeness criteria as determined by the Food Surveys Research Group (Table 1). Sample size by race/ethnicity, gender and age group was summarized and weighted to represent the US adult population of 193 million. The percentage of participants in the four age categories ranged from $11 \cdot 3 \%$ to $38 \cdot 2 \%$ for $>70$ years
Table 1 Demographic characteristics of US adults aged $\geq 19$ years, National Health and Nutrition Examination Survey, 2007-2010*,t,‡

\begin{tabular}{|c|c|c|c|}
\hline Characteristic & $n$ & $\%$ & SE \\
\hline \multicolumn{4}{|l|}{ Age group (years) } \\
\hline $19-30$ & 1820 & $21 \cdot 0$ & $0 \cdot 7$ \\
\hline $31-50$ & 3239 & $38 \cdot 2$ & 0.8 \\
\hline $51-70$ & 3025 & $29 \cdot 5$ & 0.8 \\
\hline$>70$ & 1635 & $11 \cdot 3$ & 0.4 \\
\hline \multicolumn{4}{|l|}{ Gender } \\
\hline Male & 4840 & $49 \cdot 0$ & 0.4 \\
\hline Female & 4879 & $51 \cdot 0$ & 0.4 \\
\hline \multicolumn{4}{|l|}{ BMI $\left(\mathrm{kg} / \mathrm{m}^{2}\right)$} \\
\hline$<24.9$ & 2694 & $30 \cdot 2$ & 0.8 \\
\hline $25 \cdot 0-29 \cdot 9$ & 3374 & $34 \cdot 4$ & 0.7 \\
\hline$\geq 30 \cdot 0$ & 3651 & $35 \cdot 4$ & 0.6 \\
\hline \multicolumn{4}{|l|}{ Ethnic group } \\
\hline Hispanic & 2735 & $13 \cdot 4$ & 1.9 \\
\hline Non-Hispanic White & 5005 & $74 \cdot 9$ & $2 \cdot 3$ \\
\hline Non-Hispanic Black & 1979 & $11 \cdot 8$ & $1 \cdot 2$ \\
\hline \multicolumn{4}{|l|}{ Income } \\
\hline Low & 3128 & $21 \cdot 2$ & $1 \cdot 1$ \\
\hline Middle & 1326 & $10 \cdot 6$ & 0.4 \\
\hline High & 5265 & $68 \cdot 3$ & $1 \cdot 2$ \\
\hline \multicolumn{4}{|l|}{ Vitamin D supplement } \\
\hline Yes & 2584 & $29 \cdot 7$ & 0.9 \\
\hline No & 7135 & $70 \cdot 3$ & 0.9 \\
\hline
\end{tabular}

${ }^{*}$ Total sample size of 9719 , weighted to represent the US adult population of 193 million.

tIncome categorized by poverty income ratio (PIR), with $\leq 131 \%$ of $\mathrm{PIR}=$ low income, $>131 \%$ to $\leq 185 \%$ of PIR $=$ medium income and $>185 \%$ of $\mathrm{PIR}=$ high income.

$\ddagger$ Vitamin D supplements include any supplement containing vitamin D.

and 31-50 years, respectively. The percentage of males and females included was similar $(49 \cdot 0 \%$ and $51 \cdot 0 \%$, respectively). Distribution of BMI indicated that $34 \cdot 4 \%$ were overweight $\left(\mathrm{BMI}=25 \cdot 0-29 \cdot 9 \mathrm{~kg} / \mathrm{m}^{2}\right)$ and $35 \cdot 4 \%$ were obese (BMI $\geq 30 \cdot 0 \mathrm{~kg} / \mathrm{m}^{2}$ ). The study population was comprised of $74.9 \% \mathrm{NH}$ Whites, $13.4 \%$ Hispanics and $11.8 \% \mathrm{NH}$ Blacks. The majority of the participants were in the high income group (68.3\%), although a substantial number fell into the low income category $(21 \cdot 2 \%)$ based on PIR. Approximately one-quarter (29.7\%) the participants reported consuming a supplement containing vitamin D.

\section{Estimates of vitamin D intake by income}

Mean intakes of vitamin D from food and supplements during 2007-2010 were categorized by PIR (Table 2). Total (dietary and supplemental) intake of vitamin D was significantly greater in the high income category $(>185 \%$ of PIR) $v$. the medium ( $>131 \%$ to $\leq 185 \%$ of PIR) or the low ( $\leq 131 \%$ of PIR) income categories. It should be noted, however, that the middle-income group comprised only $10.6 \%$ of the total population. Within each overall race/ethnic group when gender and age were controlled, economic status was related to differences in total, dietary and supplemental vitamin D intakes (Table 2). NH White total and supplemental vitamin D intakes of the highest income category were greater than those of the medium and low income categories. In contrast, dietary vitamin D 
Table 2 Vitamin D intakes (total, dietary and supplemental) of US adults aged $\geq 19$ years by income, gender and race/ethnicity, National Health and Nutrition Examination Survey, 2007-2010*,t,‡,§,II

\begin{tabular}{|c|c|c|c|c|c|c|c|c|c|c|c|c|c|}
\hline & \multicolumn{3}{|c|}{ Total population } & \multicolumn{3}{|c|}{ Hispanic (x) } & \multicolumn{2}{|c|}{ NH White (y) } & \multicolumn{3}{|c|}{ NH Black (z) } & \multirow[b]{2}{*}{$n$} & \multirow[b]{2}{*}{ Difference by row } \\
\hline & $\mu \mathrm{g} / \mathrm{d}$ & SE & $n$ & $\mu \mathrm{g} / \mathrm{d}$ & SE & $n$ & $\mu \mathrm{g} / \mathrm{d}$ & SE & $n$ & $\mu \mathrm{g} / \mathrm{d}$ & SE & & \\
\hline \multicolumn{14}{|l|}{ Total intake by income } \\
\hline All & $8 \cdot 6$ & 0.3 & 9719 & $8 \cdot 1$ & 0.3 & 2735 & $10 \cdot 6$ & 0.4 & 5005 & $7 \cdot 1$ & 0.3 & 1979 & $x<y, x>z, y>z$ \\
\hline Low (a) & $8 \cdot 0$ & 0.3 & 3128 & $7 \cdot 6$ & 0.5 & 1195 & $9 \cdot 4$ & 0.5 & 1320 & $6 \cdot 9$ & $0 \cdot 4$ & 613 & $y>z$ \\
\hline Medium (b) & $7 \cdot 9$ & $0 \cdot 3$ & 1326 & $8 \cdot 2$ & 0.5 & 430 & $9 \cdot 7$ & 0.7 & 561 & $5 \cdot 8$ & 0.4 & 335 & $x>z, y>z$ \\
\hline High $(c)$ & $10 \cdot 0$ & 0.3 & 5265 & $8 \cdot \overline{7}$ & 0.3 & 1110 & $12 \cdot 7$ & 0.4 & 3124 & 8.5 & 0.5 & 1031 & $x<y, y>z$ \\
\hline Difference by column & $a<c, b<c$ & & & & & & $a<c, b<c$ & & & $b<c$ & & & \\
\hline \multicolumn{14}{|l|}{ Dietary intake by income } \\
\hline All & $4 \cdot 4$ & $0 \cdot 1$ & 9719 & $4 \cdot 7$ & 0.2 & 2735 & $4 \cdot 8$ & 0.1 & 5005 & 3.5 & $0 \cdot 1$ & 1979 & $x>z, y>z$ \\
\hline Low (a) & $4 \cdot 3$ & $0 \cdot 1$ & 3128 & $4 \cdot 4$ & 0.2 & 1195 & 4.9 & 0.3 & 1320 & $3 \cdot 7$ & $0 \cdot 2$ & 613 & $y>z$ \\
\hline Medium (b) & $4 \cdot 2$ & $0 \cdot 1$ & 1326 & 4.9 & $0 . \overline{3}$ & 430 & $4 \cdot 5$ & 0.3 & 561 & $3 \cdot 0$ & 0.2 & 335 & $x>z, y>z$ \\
\hline High (c) & $4 \cdot 6$ & $0 \cdot 1$ & 5265 & $4 \cdot 8$ & 0.2 & 1110 & $5 \cdot 1$ & $0 \cdot 1$ & 3124 & $4 \cdot 0$ & $0 \cdot 2$ & 1031 & $y>z$ \\
\hline Difference by column & $b<c$ & & & & & & & & & $b<c$ & & & \\
\hline \multicolumn{14}{|c|}{ Supplemental intake by income } \\
\hline All & $4 \cdot 3$ & 0.3 & 9719 & $3 \cdot 4$ & 0.3 & 2736 & $5 \cdot 8$ & 0.3 & 5005 & 3.6 & 0.3 & 1979 & $x<y, y>z$ \\
\hline Low (a) & $3 \cdot 7$ & $0 \cdot 2$ & 3128 & $3 \cdot 2$ & 0.4 & 1196 & $4 \cdot 5$ & 0.4 & 1320 & $3 \cdot 3$ & $0 \cdot 4$ & 613 & \\
\hline Medium (b) & $3 \cdot 8$ & $0 \cdot 3$ & 1326 & $3 \cdot 3$ & 0.5 & 430 & $5 \cdot 2$ & 0.6 & 561 & $2 \cdot 8$ & $0 \cdot 4$ & 335 & \\
\hline High (c) & $5 \cdot 4$ & 0.3 & 5265 & 3.9 & 0.3 & 1110 & $7 \cdot \overline{7}$ & 0.4 & 3124 & $4 \cdot 5$ & $0 \cdot 4$ & 1031 & $x<y, y>z$ \\
\hline Difference by column & $\mathrm{a}<\mathrm{c}, \mathrm{b}<\mathrm{c}$ & & & & & & $\mathrm{a}<\mathrm{c}, \mathrm{b}<\mathrm{c}$ & & & $\mathrm{b}<\mathrm{c}$ & & & \\
\hline \multicolumn{14}{|l|}{ Total intake by gender } \\
\hline Male (a) & $8 \cdot 1$ & $0 \cdot 2$ & 4840 & $8 \cdot 1$ & 0.4 & 1340 & $9 \cdot 8$ & 0.5 & 2526 & $6 \cdot 6$ & $0 \cdot 4$ & 974 & $x<y, y>z$ \\
\hline Female (b) & $9 \cdot 1$ & $0 . \overline{3}$ & 4879 & $7 \cdot 7$ & 0.3 & 1395 & $11 \cdot 8$ & 0.6 & 2479 & $7 \cdot 6$ & 0.4 & 1005 & $x<y, y>z$ \\
\hline Difference by column & $a<b$ & & & & & & & & & & & & \\
\hline \multicolumn{14}{|l|}{ Dietary intake by gender } \\
\hline Male (a) & $4 \cdot 9$ & $0 \cdot 1$ & 4840 & $5 \cdot 1$ & 0.2 & 1340 & $5 \cdot 6$ & 0.2 & 2526 & $3 \cdot 9$ & 0.2 & 974 & $x>z, y>z$ \\
\hline Female (b) & $3 \cdot 8$ & $0 \cdot 1$ & 4879 & $4 \cdot 1$ & 0.2 & 1395 & $4 \cdot 0$ & $0 \cdot 1$ & 2479 & $3 \cdot 2$ & 0.2 & 1005 & $y>z$ \\
\hline Difference by column & $a>b$ & & & $a>b$ & & & $a>b$ & & & & & & \\
\hline \multicolumn{14}{|c|}{ Supplemental intake by gender } \\
\hline Male (a) & $3 \cdot 3$ & 0.2 & 4840 & $3 \cdot 0$ & 0.3 & 1340 & $4 \cdot 1$ & 0.4 & 2526 & $2 \cdot 7$ & 0.3 & 974 & $y>z$ \\
\hline Female (b) & $5 \cdot 3$ & 0.2 & 4879 & 3.6 & 0.3 & 1395 & $7 \cdot 8$ & 0.5 & 2479 & $4 \cdot 4$ & 0.3 & 1005 & $x<y, y>z$ \\
\hline Difference by column & $a<b$ & & & & & & $a<b$ & & & $a<b$ & & & \\
\hline
\end{tabular}

$\mathrm{NH}$, Non-Hispanic.

*Values are means with their standard errors.

tIncome categorized by poverty income ratio (PIR), with $\leq 131 \%$ of PIR $=$ low income, $>131 \%$ to $\leq 185 \%$ of $\mathrm{PIR}=\mathrm{medium}$ income and $>185 \%$ of $\mathrm{PIR}=$ high income.

¥Multiple comparisons by PIR were adjusted for age and gender. Tukey-Kramer adjustment was used for control for the family-wise error.

§Multiple comparisons by gender were adjusted for age and PIR. Tukey-Kramer adjustment was used for control for the family-wise error

IISignificant differences by row or column groups indicated by letter: $P<0.05$.

intake of $\mathrm{NH}$ Whites did not vary by income status. Likewise, NH Black total vitamin D intake was greater in the high $v$, the medium income category, but not compared with the low income category. High-income $\mathrm{NH}$ Blacks also had higher dietary and supplemental vitamin D intakes than medium-income NH Blacks. Surprisingly, within the Hispanic group, income status was not associated with differences in vitamin D intake.

\section{Estimates of vitamin D intake by race/etbnicity}

When vitamin D intake (total, dietary and supplemental) was compared for all participants in race/ethnic groups, NH Whites had a significantly higher intake of vitamin D than NH Blacks and Hispanics (Table 2). Vitamin D intake (total, dietary and supplemental) by Hispanics was also higher than in NH Blacks.

\section{Estimates of vitamin $D$ intake by income and race/ethnicity}

When groups were compared by income category across race/ethnicity, total vitamin $\mathrm{D}$ intake of $\mathrm{NH}$ Whites was greater than that of Hispanics and $\mathrm{NH}$ Blacks within the high income category (Table 2). Dietary intake by $\mathrm{NH}$ Whites was higher than that by NH Blacks for all income levels. Distinctions by income category of supplemental vitamin D intake between race/ethnic groups were apparent only for the high income category, with $\mathrm{NH}$ Whites having greater supplemental vitamin D intake than Hispanics and NH Blacks.

\section{Estimates of vitamin $D$ intake by gender}

For the population as a whole, gender influenced total vitamin D intake. Although supplemental vitamin D intake was significantly higher for all females than for all males (Table 2), dietary intake of vitamin $\mathrm{D}$ by females was less. When vitamin D intake was examined across gender and race/ethnic groups, significant differences were apparent for females. Total and supplemental vitamin $\mathrm{D}$ intakes of $\mathrm{NH}$ White females were greater than those of NH Black females and Hispanic females. Dietary vitamin $\mathrm{D}$ intake of females, however, was significantly higher for $\mathrm{NH}$ Whites compared with NH Black females, 
Table 3 Percentage of US adults aged $\geq 19$ years not meeting the EAR and the RDA for vitamin D by income and age, National Health and Nutrition Examination Survey, 2007-2010*,t,‡

\begin{tabular}{|c|c|c|c|c|c|c|c|c|c|}
\hline & \multirow[b]{2}{*}{$n$} & \multicolumn{2}{|c|}{$\%$ not meeting the EAR } & \multicolumn{2}{|c|}{$\%$ not meeting the EAR } & \multicolumn{2}{|c|}{$\%$ not meeting the RDA } & \multicolumn{2}{|c|}{$\%$ not meeting the RDA } \\
\hline & & By total intake & SE & By dietary intake & $\mathrm{SE}$ & By total intake & $\mathrm{SE}$ & By dietary intake & $\mathrm{SE}$ \\
\hline \multicolumn{10}{|l|}{ Income } \\
\hline All & 9725 & $64 \cdot 6$ & $1 \cdot 0$ & $89 \cdot 1$ & 0.5 & $78 \cdot 8$ & $0 \cdot 8$ & $96 \cdot 2$ & 0.3 \\
\hline Low & 3131 & $75 \cdot 2$ & $1 \cdot 1$ & $90 \cdot 0$ & 0.7 & $86 \cdot 8$ & $0 \cdot 8$ & $96 \cdot 5$ & 0.5 \\
\hline Medium & 1326 & $71 \cdot 2$ & $2 \cdot 4$ & $91 \cdot 2$ & $1 \cdot 3$ & $84 \cdot 3$ & $2 \cdot 0$ & $97 \cdot 0$ & 0.6 \\
\hline High & 5266 & $59 \cdot 9$ & $1 \cdot 2$ & $88 \cdot 5$ & 0.6 & $75 \cdot 4$ & $1 \cdot 0$ & $96 \cdot 2$ & 0.3 \\
\hline \multicolumn{10}{|c|}{ Age (years) } \\
\hline All & 10780 & $64 \cdot 7$ & 0.9 & $89 \cdot 1$ & 0.5 & $78 \cdot 8$ & $0 \cdot 8$ & $96 \cdot 2$ & 0.3 \\
\hline $19-30$ & 2038 & $76 \cdot 8$ & $1 \cdot 3$ & $88 \cdot 4$ & $1 \cdot 0$ & $89 \cdot 5$ & $1 \cdot 1$ & $96 \cdot 5$ & 0.6 \\
\hline $31-50$ & 3547 & $71 \cdot 2$ & $1 \cdot 2$ & $89 \cdot 0$ & $1 \cdot 0$ & $83 \cdot 5$ & $1 \cdot 0$ & $96 \cdot 2$ & 0.5 \\
\hline $51-70$ & 3379 & $53 \cdot 7$ & $1 \cdot 4$ & $88 \cdot 6$ & $0 \cdot 8$ & $66 \cdot 7$ & $1 \cdot 4$ & $95 \cdot 0$ & 0.6 \\
\hline$>70$ & 1816 & $48 \cdot 5$ & $2 \cdot 0$ & $91 \cdot 7$ & $0 \cdot 7$ & $74 \cdot 2$ & $1 \cdot 9$ & $98 \cdot 8$ & 0.3 \\
\hline
\end{tabular}

EAR, Estimated Average Requirement.

*Values are mean percentages and their standard errors.

tEAR $=10 \mu \mathrm{g} / \mathrm{d} ; \mathrm{RDA}=16 \mu \mathrm{g} / \mathrm{d}$ for $19-70$ years and $20 \mu \mathrm{g} / \mathrm{d}$ for $>70$ years

flncome categorized by poverty income ratio (PIR), with $\leq 131 \%$ of $\mathrm{PIR}=$ low income, $>131 \%$ to $\leq 185 \%$ of $\mathrm{PIR}=\mathrm{medium}$ income and $>185 \%$ of $\mathrm{PIR}=$ high income.

but not Hispanic females. In contrast, total vitamin D intake of NH White males was higher than that of both NH Black and Hispanic males.

\section{Vitamin $D$ supplement use by income, gender and etbnicity}

Participants within the high income category (33.1\%) were more likely to consume supplements that contained vitamin $\mathrm{D}$ than participants within the medium income $(22.5 \%)$ or low income $(17 \cdot 6 \%)$ categories (data not shown). In addition, females $(30 \cdot 1 \%)$ were more likely than males $(23.0 \%)$ to report taking supplements containing vitamin D. Furthermore, more NH Whites (34.7\%) than Hispanics $(16 \cdot 2 \%)$ or NH Blacks $(20 \cdot 0 \%)$ consumed supplements containing vitamin D.

\section{Percentage not consuming the Estimated Average Requirement or the RDA by income and age}

A substantial percentage $(64 \cdot 6 \%)$ of the US population of adults in 2007-2010 did not meet the EAR for vitamin D (Table 3). When examined by income category, the percentage not meeting the EAR for vitamin D by total intake (diet and supplements) was less in the high income category $(59.9 \%) v$. the low income category $(75 \cdot 2 \%)$. The percentage not meeting the EAR for vitamin $\mathrm{D}$ by dietary intake showed little variation by income category.

Examining the percentage not meeting the EAR for vitamin $\mathrm{D}$ for total intake (diet plus supplements) by age indicated that the lowest percentages not meeting the EAR were in the older age groups $(53.7 \%$ of $51-70$ year group and $48.5 \%$ of $>70$ year group; Table 3 ). The percentage not meeting the EAR for vitamin D by diet alone was high (88.4-91.7\%) and showed little variation among age groups. Therefore, use of supplements was largely responsible for decreasing the percentage of participants not meeting the EAR with ageing.
A high percentage (78.8\%) of adults did not meet the RDA for vitamin D (Table 3). When examined by income, the percentage not meeting the RDA for vitamin D by total intake (diet and supplements) was less in the high $(75 \cdot 4 \%) v$. the medium $(84 \cdot 3 \%)$ or the low $(86 \cdot 8 \%)$ income categories. Moreover, the percentage not meeting the RDA by total intake (diet plus supplements) was less for adults aged $51-70$ years $(66.7 \%)$ and $>70$ years $(74 \cdot 2 \%)$. Again, use of supplements largely explained the decreasing percentage of participants not meeting the RDA with ageing.

\section{Estimates of vitamin $D$ contributions from food and supplement sources}

Fortified milk and milk products provided the greatest contribution $(43.7 \%)$ to the dietary vitamin D intake in the USA (Table 4). Other major food group sources of vitamin $\mathrm{D}$ were meat and fish $(25 \cdot 8 \%)$, grain products $(12 \cdot 2 \%)$, and to a lesser extent eggs and egg dishes (9.5\%) and fortified fruit juices $(2 \cdot 8 \%)$. When the total food categories were assessed by income, the high income category consumed the greatest proportion of the total vitamin $\mathrm{D}$ intake of the population; however, the high-income group (68.3\%) also represented the largest proportion of the population. Nevertheless, a higher proportion of vitamin D intake from milk and milk products $(35 \cdot 8 \%)$ intake was consumed by the lower income group which represented only $21 \cdot 2 \%$ of the sample population.

\section{Discussion}

To our knowledge, the present study is the first to examine US disparities of vitamin D intake by income status during 2007-2010. Total (dietary plus supplemental) intake of vitamin D in the US population was significantly greater in the high income category $v$. the 
Table 4 Proportion of vitamin D intake from major dietary sources for US adults aged $\geq 19$ years, National Health and Nutrition Examination Survey, 2007-2010*, +

\begin{tabular}{|c|c|c|c|c|c|}
\hline \multirow[b]{2}{*}{ Food category } & \multirow{2}{*}{$\begin{array}{l}\% \text { of total } \\
\text { intake }\end{array}$} & \multirow{2}{*}{$\begin{array}{l}\% \text { within } \\
\text { category }\end{array}$} & \multicolumn{3}{|c|}{ Proportion of total category by income (\%) } \\
\hline & & & Low & Medium & High \\
\hline Eggs & $9 \cdot 5$ & & $34 \cdot 0$ & $9 \cdot 3$ & $55 \cdot 6$ \\
\hline Fruit and Juices & $2 \cdot 8$ & & $36 \cdot 5$ & $11 \cdot 8$ & $52 \cdot 1$ \\
\hline Grains & $12 \cdot 2$ & & $34 \cdot 3$ & $12 \cdot 9$ & $52 \cdot 8$ \\
\hline Cereals & & $5 \cdot 6$ & & & \\
\hline Grain Mixtures & & $3 \cdot 3$ & & & \\
\hline Milk and Milk Products & $43 \cdot 7$ & & $35 \cdot 8$ & $12 \cdot 8$ & $51 \cdot 4$ \\
\hline Milk and Milk Drinks & & $39 \cdot 2$ & & & \\
\hline Cheese & & $4 \cdot 0$ & & & \\
\hline Meats and Fish & $25 \cdot 8$ & & $27 \cdot 0$ & $10 \cdot 3$ & $62 \cdot 7$ \\
\hline Fish and Shellfish & & $12 \cdot 1$ & & & \\
\hline Meat, Poultry, Fish with Non-meat Items & & $5 \cdot 8$ & & & \\
\hline Organ Meat, Sausage, Lunchmeats, Meat Spreads & & $3 \cdot 1$ & & & \\
\hline Pork & & $2 \cdot 1$ & & & \\
\hline Beef & & $1 \cdot 0$ & & & \\
\hline Poultry & & 0.7 & & & \\
\hline
\end{tabular}

*Food categories based on the US Department of Agriculture's Food and Nutrient Database for Dietary Surveys food groups.

tProportion of total category by income categorized by poverty income ratio (PIR), with $\leq 131 \%$ of PIR $=$ low income, $>131 \%$ to $\leq 185 \%$ of PIR $=$ medium income and $>185 \%$ of PIR $=$ high income.

medium and low income categories. Previously, dietary intake of vitamin D and serum 25(OH)D concentrations from NHANES III and NHANES 1999-2000 were also compared with PIR ${ }^{(34)}$. PIR was categorized as poor, near poor or not poor; however, PIR was not a predictor of dietary intake of vitamin $\mathrm{D}$ and, surprisingly, marginal serum $25(\mathrm{OH}) \mathrm{D}$ concentrations were more likely to occur at higher PIR ${ }^{(34)}$.

The effect of income on vitamin $\mathrm{D}$ intake during 2007-2010 was more complex when examined within specific race/ethnic groups. Although $\mathrm{NH}$ Whites and Hispanics in the highest income category had greater combined total and supplemental vitamin D intakes than those in the medium and low income categories, $\mathrm{NH}$ Blacks in the high income category differed only from the medium-income group. In contrast, Hispanics' income status was not associated with differences in total, dietary or supplemental vitamin D intake. Only $16 \cdot 2 \%$ of Hispanics consumed supplements containing vitamin $\mathrm{D}$, and therefore total vitamin $\mathrm{D}$ intake was more dependent on dietary sources.

Overall, the greatest total vitamin $\mathrm{D}$ intake was reported by high-income NH Whites $(12 \cdot 7$ (SE 0.4$) \mu \mathrm{g} / \mathrm{d}$ ) with a PIR of $>185 \%$, and the lowest total dietary vitamin D intake was reported by medium-income $\mathrm{NH}$ Blacks $(5 \cdot 8$ (SE $0 \cdot 4) \mu \mathrm{g} / \mathrm{d}$ ) with a PIR of $>131 \%$ to $\leq 185 \%$. In the lower income category ( $\leq 131 \%$ of PIR) the additional expense of consuming dietary supplements likely limited the use of vitamin D supplements for all ethnic groups. Previously, differences in vitamin D intake were also reported between race/ethnic groups ${ }^{(35,36)}$. NHANES estimated intakes of vitamin D from food and supplements from 1999-2000 were consistently lower for $\mathrm{NH}$ Blacks compared with $\mathrm{NH}$ Whites, but not compared with Mexican Americans (similar to 2007-2010 NHANES
Hispanics) ${ }^{(35)}$. Likewise, earlier NHANES III (1988-1994) daily vitamin D intakes by African Americans (similar to 2007-2010 NHANES NH Blacks) were significantly lower than those by $\mathrm{NH}$ Whites and Mexican Americans ${ }^{(36)}$.

Over the last 30 years a trend is apparent in the USA of increased total vitamin D intake (diet plus supplements) by all race/ethnic groups. Mean intake of vitamin $\mathrm{D}$ of adults $\geq 19$ years from the NHANES 1998-1994 survey was in the range of $7 \cdot 33-8 \cdot 37 \mu \mathrm{g} / \mathrm{d}$ for Whites, $5 \cdot 73-6.90 \mu \mathrm{g} / \mathrm{d}$ for African Americans and 5.69-6.16 $\mu \mathrm{g} / \mathrm{d}$ for Mexican Americans ${ }^{(36)}$; vitamin $\mathrm{D}$ intake from NHANES $1999-2000$ ranged from $7 \cdot 8$ to $10 \cdot 3 \mu \mathrm{g} / \mathrm{d}$ for $\mathrm{NH}$ Whites, from $5 \cdot 3$ to $6 \cdot 1 \mu \mathrm{g} / \mathrm{d}$ for $\mathrm{NH}$ Blacks and from $5 \cdot 7$ to $6.9 \mu \mathrm{g} / \mathrm{d}$ Mexican Americans ${ }^{(35)}$; and in the present analysis the total vitamin D intake in 2007-2010 was higher than in previous years and averaged $10.6 \mu \mathrm{g} / \mathrm{d}$ for $\mathrm{NH}$ Whites, $7 \cdot 1 \mu \mathrm{g} / \mathrm{d}$ for $\mathrm{NH}$ Blacks and $8 \cdot 1 \mu \mathrm{g} / \mathrm{d}$ for Hispanics (Table 2).

Dietary intake and use of supplements continue to make an important contribution to vitamin D status despite potential racial and ethnic differences in cutaneous synthesis of vitamin D and supplementation practices ${ }^{(6)}$. In previous studies with $\mathrm{NH}$ Blacks and $\mathrm{NH}$ Whites, a lack of vitamin D supplementation increased the odds of a vitamin $\mathrm{D}$ insufficiency ${ }^{(19,37)}$. In the present study, vitamin D supplementation differed by gender and race/ethnicity (Table 2). Vitamin D intake from supplements was higher for all females $(5.3(\operatorname{se~} 0.2) \mu \mathrm{g} / \mathrm{d})$ than for all males $(3.3$ (SE $0 \cdot 2) \mu \mathrm{g} / \mathrm{d}$ ). NH White females' intake from supplements containing vitamin D ( $7 \cdot 8($ SE 0.5$) \mu \mathrm{g} / \mathrm{d})$ was nearly double that of $\mathrm{NH}$ Black females (4.4 (SE $0 \cdot 3) \mu \mathrm{g} / \mathrm{d}$ ) and was more than double the intake of Hispanic females (3.6 (SE 0.3$) \mu \mathrm{g} / \mathrm{d})$. Similarly, intake of vitamin D from supplements by NH White males $(4 \cdot 1($ se $0 \cdot 4) \mu \mathrm{g} / \mathrm{d}$ ) was approximately $37-52 \%$ higher than that by Hispanic males 
$(3 \cdot 0(\operatorname{se} 0 \cdot 3) \mu \mathrm{g} / \mathrm{d})$ and NH Black males $(2 \cdot 7(\operatorname{SE} 0 \cdot 3) \mu \mathrm{g} / \mathrm{d})$, respectively.

Obtaining sufficient vitamin D from food sources is challenging. Although consumption of fluid milk has shown a downward trend, recently more vitamin D-fortified products (yoghurts, margarine brands, cereal bars, etc.) have emerged in the marketplace to provide new sources of vitamin $\mathrm{D}^{(13)}$. Despite the existence of more fortified foods in the marketplace, fortified milk provided the greatest source of vitamin D in the US diet in 2007-2010 (43.7\%). Overall, fortified foods from several food groups provided $\sim 62 \%$ of the vitamin D intake from food, clearly indicating that consumption of fortified foods played a critical role in the dietary intake of this essential vitamin. Nevertheless, use of fortified milk differs among racial groups in the USA and barriers include food preferences, cultural tradition and cost $^{(38)}$. Groups in greatest need of increasing vitamin D intake consume significantly lower amounts of commonly fortified foods such as milk and ready-to-eat cereals. Although vitamin D-fortified milk is relatively inexpensive per serving, the cost of plant-based milk and enriched mushrooms containing vitamin D may prevent lower economic groups from choosing these foods ${ }^{(38)}$. Moreover, a systematic review of nine clinical trials found that fortification of a food with vitamin $\mathrm{D}$ was associated with statistically significant improvements in serum 25(OH)D concentrations, which has important implications for the maintenance of vitamin D status in the population ${ }^{(39)}$.

Major strengths and limitations of the present study should be noted. The primary strength was the large sample size and population-based analyses of vitamin D intakes over a period of 4 years. In addition, the vitamin D content of foods of the National Nutrient Database was largely based on new analytical data and not imputed data. Furthermore, with the establishment of an EAR and RDA for vitamin D in 2010, intakes by income categories and age could be compared. Nevertheless, this was a cross-sectional study and therefore causal inferences cannot be made. In addition, use of one $24 \mathrm{~h}$ dietary recall is dependent on memory, may not reflect usual intake and be subject to recall errors and under-reporting ${ }^{(40)}$; however, a single $24 \mathrm{~h}$ recall is sufficient to report mean usual intake of a group ${ }^{(41)}$.

\section{Conclusions}

Our results identified greater total intake of vitamin D at higher income levels. Healthy People 2010 and 2020 include eliminating health disparities in the USA as an overarching goal $^{(42)}$. It is conceivable that differences in vitamin D intake are contributing to observed health status disparities in the USA. In a national survey, socio-economic status explained a considerable portion of the nutritional and health differences between races/ethnicities. Socio-economic status was associated with $30 \%$ of the black-white difference in dietary quality and approximately $40 \%$ of the prevalence of overweight and obesity ${ }^{(43)}$. Recently, after controlling for covariates and socio-economic factors, disparities in $25(\mathrm{OH}) \mathrm{D}$ concentrations related to ethnic skin colour were considered to be an important biological determinant of US health disparities ${ }^{(23)}$.

The 2010 Dietary Guidelines for Americans identified low vitamin D intake as a public health concern ${ }^{(44)}$. Encouraging the consumption of foods naturally high in vitamin $\mathrm{D}$ and foods fortified with vitamin $\mathrm{D}$, along with supporting the greater use of dietary supplements, would help to improve vitamin D status in the USA. Finally, culture-specific interventions are needed to increase vitamin D supplement use and health messages should be specifically targeted to all males, Blacks and Hispanics.

\section{Acknowledgements}

Sources of funding: This project was funded by a Chancellor's Research Fellowship from Texas Woman's University Office of Research \& Sponsored Programs. The Texas Woman's University Office of Research \& Sponsored Programs had no role in the design, analysis or writing of this article. Conflicts of interest: C.E.M., J.D.R. and Y.L. have no conflicts of interest to report. Ethics: This study was approved by the Institutional Review Board of Texas Woman's University, Houston Center, TX, USA. Author' contributions: C.E.M., J.D.R. and Y.L. designed the study; Y.L. performed the data analysis; C.E.M. wrote the manuscript and had primary responsibility for the final content; J.D.R. and Y.L. edited the manuscript.

\section{References}

1. Manson JE, Bassuk SS, Lee I et al. (2012) The Vitamin D and OmegA Trial (VITAL): rationale and design of a large randomized controlled trial of vitamin $\mathrm{D}$ and marine omega-3 fatty acid supplements for the primary prevention of cancer and cardiovascular disease. Contemp Clin Trials 33, 159-171.

2. Parker J, Hashmi O, Dutton D et al. (2010) Levels of vitamin $\mathrm{D}$ and cardiometabolic disorders: systematic review and meta-analysis. Maturitas 65, 225-236.

3. Golden SH, Brown A, Cauley JA et al. (2012) Health disparities in endocrine disorders: biological, clinical, and clinical factors - an Endocrine Society scientific statement. $J$ Clin Endocrinol Metab 97, E1579-E1639.

4. Forrest KYZ \& Stuhldreher WL (2011) Prevalence and correlates of vitamin D deficiency in US adults. Nutr Res 31, $48-54$.

5. Grant WB \& Peiris AN (2010) Possible role of serum 25-hydroxyvitamin D in Black-White health disparities in the United States. J Am Med Dir Assoc 11, 617-628.

6. Fulgoni VL, Keast DR, Bailey RL et al. (2011) Foods, fortificants, and supplements: where do Americans get their nutrients? J Nutr 141, 1847-1854.

7. Bailey RL, Dodd KW, Goldman JA et al. (2010) Estimation of total usual calcium and vitamin D intakes in the United States. J Nutr 140, 817-822.

8. Holick MF (2007) Vitamin D deficiency. N Engl J Med 357, 266-281. 
9. Yetley EA (2008) Vitamin D nutritional status of US population. Am J Clin Nutr 88, issue 2, 558S-564S.

10. Holden JM \& Lemar LE (2008) Assessing vitamin D contents in foods and supplements: challenges and needs. Am J Clin Nutr 88, issue 2, 551S-553S.

11. Byrdwell WC, DeVires J, Exler J et al. (2008) Analyzing vitamin $\mathrm{D}$ in foods and supplements: methodologic challenges. Am J Clin Nutr 88, issue 2, 554S-557S.

12. Patterson KY, Phillips KM, Horst RL et al. (2010) Vitamin D content and variability in fluid milks from a US Department of Agriculture nationwide sampling to update values in the National Nutrient Database for Standard Reference. J Dairy Sci 93, 5082-5090.

13. Harnack LJ, Steffen L, Zhou X et al. (2011) Trends in vitamin $\mathrm{D}$ intake from food sources among adults in the Minneapolis-St Paul, MN, metropolitan area, 1980-1982 through 2007-2009. J Acad Nutr Diet 111, 1329-1334.

14. Institute of Medicine, Food and Nutrition Board (1997) Dietary Reference Intakes for Calcium, Phosphorus, Magnesium, Vitamin D and Fluoride. Washington, DC: National Academies Press.

15. Institute of Medicine, Food and Nutrition Board (2010) Dietary Reference Intakes for Calcium and Vitamin D. Washington, DC: National Academy Press.

16. Harris SS \& Dawson-Hughes B (1998) Seasonal changes in plasma 25-hydroxyvitamin D concentrations of young American black and white women. Am J Clin Nutr 67, $1232-1236$.

17. Harris SS, Soteriades E, Coolidge JA et al. (2000) Vitamin D insufficiency and hyperparathyroidism in low income, multiracial, elderly population. J Clin Endocrinol Metab 85, 4125-4130.

18. Looker AC, Dawson-Hughes B, Calvo MS et al. (2002) Serum 25-hydroxyvitamin D status of adolescents and adults in two seasonal subpopulation from NHANES III. Bone 30, 771-777.

19. Nesby-O'Dell S, Scanlon KS, Cogswell ME et al. (2002) Hypovitaminosis D prevalence and determinants among African American and white women of reproductive age: third National Health and Nutrition Examination Survey. 1988-1884. Am J Clin Nutr 76, 187-192.

20. Zadshir A, Tareen N, Pan D et al. (2005) The prevalence of hypovitaminosis D among US adults: data from the NHANES III. Ethn Dis 55, 97-101.

21. Bleich SN, Thorpe RJ, Sharif-Harris H et al. (2010) Social context explains race disparities in obesity among women. J Epidemiol Community Health 64, 465-469.

22. American Academy of Nutrition and Dietetics (2011) Practice paper of the American Academy of Nutrition and Dietetics: addressing racial and ethnic health disparities. $J$ Acad Nutr Diet 111, 446-456.

23. Weishaar T \& Marcley Vergili J (2013) Vitamin D statues is a biological determinant of health disparities. J Acad Nutr Diet 113, 643-651.

24. Clemens TL, Henderson SL, Adams JS et al. (1982) Increased skin pigment reduces the capacity of skin to synthesize vitamin $\mathrm{D}_{3}$. Lancet $\mathbf{1}, 74-76$.

25. National Center for Health Statistics, Centers for Disease Control and Prevention (2013) About the National Health and Nutrition Examination Survey. http://www.cdc.gov/ nchs/nhanes/about_nhanes.htm (accessed October 2013).

26. National Center for Health Statistics, Centers for Disease Control and Prevention (2011) National Health and Nutrition Examination Survey, Analytic note regarding 2007-2010 survey design changes and combining data across other survey cycles. http://www.cdc.gov/nchs/data/ nhanes/analyticnote_2007-2010.pdf (accessed November 2012).

27. Blanton CA, Moshfegh AJ, Baer DJ et al. (2006) The USDA automated multiple-pass method accurately estimates group total energy and nutrient intake. J Nutr 136, 2594-2599.

28. National Center for Health Statistics, Centers for Disease Control and Prevention (2010) 2009-2010 National Health and Nutrition Examination Survey (NHANES) Survey Operations Manuals, Brochures, and Consent Documents. http://www.cdc.gov/nchs/nhanes/nhanes2009-2010/current_ nhanes_09_10.htm (accessed November 2012).

29. National Center for Health Statistics, Centers for Disease Control and Prevention (2010) National Health and Nutrition Examination. Data documentation, codebook, and frequencies. http://www.cdc.gov/nchs/nhanes/nhanes2007-2008/ DRXDOC_E.htm\#Analytic_Notes (accessed November 2012).

30. National Center for Health Statistics, Centers for Disease Control and Prevention (2010) National Health and Nutrition Examination. Survey questionnaires, examination components and laboratory components 2009-2010. Screener modules. http://www.cdc.gov/nchs/nhanes/nhanes2009-2010/ questexam09_10.htm (accessed November 2012).

31. US Census Bureau (2013) How the Census Bureau measures poverty. http://www.census.gov/hhes/www/poverty/about/ overview/measure.html (accessed October 2013).

32. National Center for Health Statistics, Centers for Disease Control and Prevention (2008) National Health and Nutrition Examination. National Health and Nutrition Examination Survey MEC In-Person Dietary Interviewers Procedure Manual. http://www.cdc.gov/nchs/data/nhanes/ nhanes_07_08/manual_dietarymec.pdf (accessed November 2012).

33. US Department of Agriculture, Agricultural Research Service (2013) Food and nutrient database for dietary studies. http://www.ars.usda.gov/services/docs.htm?docid= 12089 (accessed October 2013).

34. Kant AK \& Graubard BI (2007) Ethnicity is an independent correlate of biomarkers of micronutrient intake and status in American adults. J Nutr 137, 2456-2463.

35. Moore CE, Murphy MM \& Holick MF (2005) Vitamin D intakes by children and adults in the United States differ among ethnic groups. J Nutr 35, 2478-2485.

36. Calvo MS, Whiting SJ \& Barton CN (2004) Vitamin D fortification in the United States and Canada: current status and data needs. Am J Clin Nutr 80, 6 Suppl., 1710S-1716S.

37. Shea MK, Houston DK, Tooze JA et al. (2011) Correlates and prevalence of insufficient 25-hydroxyvitamin D status in black and white older adults: the Health ABC Study. $J$ Am Geriatr Soc 59, 1165-1174.

38. Calvo MS \& Whiting SJ (2013) Survey of current vitamin D food fortification practices in the United States and Canada. J Steroid Biochem Mol Biol 136, 1904-1911.

39. O'Donnell S, Cranney A, Horsley T et al. (2008) Efficacy of food fortification on serum 25-hydroxyvitamn D concentrations: systematic review. Am J Clin Nutr 88, $1528-1534$.

40. Willett W (1998) Nutritional Epidemiology, 2nd ed. New York: Oxford University Press.

41. Thompson FE \& Byers T (1994) Dietary assessment resource manual. J Nutr 124, 11 Suppl., 2245S-2317S.

42. Braveman PA, Kumanyika S, Fielding J et al. (2011) Health disparities and health equity: the issue is justice. $\mathrm{Am} \mathrm{J}$ Public Health 202, Suppl. 1, S149-S155.

43. Wang Y \& Chen X (2011) How much of racial/ethnic disparities in dietary intakes, exercise, and weight status can be explained by nutrition- and health-related psychosocial factors and socioeconomic status among US adults? J Acad Nutr Diet 111, 1904-1911.

44. US Department of Health and Human Services \& US Department of Agriculture (2010) Dietary Guidelines for Americans 2010. http://www.cnpp.usda.gov/Publications/ DietaryGuidelines/2010/PolicyDoc/PolicyDoc.pdf (accessed September 2013). 